\title{
Strengthening Enterprises Growth and Effectiveness in Developing Economies. A case of Very Small, Small and Medium-sized Enterprises in Cameroon
}

\author{
Bertrand Aimé Bidja ${ }^{1}$, Kudzai Mandizvidza ${ }^{2}$ \\ ${ }^{1}$ (PHD Candidate University of International Business and Economics (UIBE), Beijing, China \\ (aimebert@yahoo.fr)) \\ 2 (Development Economist and International Consultant (kudzaimandizvidza@outlook.com))
}

\begin{abstract}
The objective of this research was to apply scientific methodology to identify the factors that affect the growth and effectiveness of Very Small, Small and Medium-sized Enterprises (SMEs) in Cameroon. This was done through analyzing data on a sample which was randomly selected from two purposively chosen Cameroonian cities, Douala and Yaounde. A structured questionnaire was used for data gathering on entrepreneur characteristics as well as the internal and external factors that were assumed to have possible impact on SME growth and development. The reliability or internal consistency of the data set was determined by means of the Cronbach's Alpha procedure. The factors that have an impact on the SME growth and effectiveness were empirically analyzed by means of the Pearson's Correlation coefficient approach. The study concludes that the factors that are imperative in the success of SMEs are generally of a socio-economic as well as a political nature. A package of recommendations was suggested to facilitate the enhancement of SMEs in Cameroon as well as similar developing countries.
\end{abstract}

Keywords: Cameroon, Effectiveness, Growth, Medium-sized Enterprises, Small Enterprises, Very Small Enterprises

\section{Introduction}

There has been a progression of the world towards a knowledge-based economy that is driven by rapidly changing technologies and markets (Doh and Acs, 2010). It has accordingly remained a central agenda for policymakers around the world to direct the developing economies onto a growth path that aims to ensure sustainable economic development. Entrepreneurship has persisted as one of the important drivers of sustainable economic growth and development in the developing world. The role of SMEs in bringing forth this development can also not be underestimated since this sector, for instance in Cameroon, officially constitutes 95\% of the economy as confirmed by the Ministry of SMEs, Social Economy and Craft (Business In Cameroon, 2015). SMEs also provide the sources for most innovations, industrial renewal, job creation, export growth, and productivity (Doh and Kim, 2014). Their performance as well as the factors that affect their growth therefore deserves attention. Regardless of them being viewed as the engines of growth in Cameroon, SMEs nevertheless make up only $36 \%$ of Cameroon's GDP. It becomes crucial hence to investigate into possible means to boost the performance of this vital sector.

\section{Research Objective}

The aim of this study was to scientifically identify the factors that affect the growth and effectiveness of Very Small, Small and Medium-sized Enterprises in Cameroon. The ultimate goal is to offer recommendations for the enhancement of the sub-sector in the developing world.

\section{Research Hypotheses}

a) Entrepreneur characteristics have no impact on the SME's growth and effectiveness in Cameroon,

b) There are no internal and external factors that affect the growth and effectiveness of SMEs in Cameroon.

\section{Significance Of Study}

The empirical investigation of the distinct factors that impact the growth and effectiveness of SMEs in Cameroon remains substantial and is of paramount interest to entrepreneurs, economic development agencies, policy makers and other economic stakeholders. This study proffers some pertinent insights on the important socio-economic and political issues that relevant economic players should pay attention to in promoting the growth and effectiveness of the SMEs in Cameroon and the rest of the developing world. 


\section{Role of SMEs In Developing Countries}

SMEs have generally been regarded as increasingly important in many economies due to their crucial role in the performance of the nation through employment creation, wealth creation, as well as innovation development. They have also been considered to as engines of growth, nucleus of any economy and their great contribution to social cohesion, poverty alleviation and local and regional development has not gone unacknowledged. (Kamunge, Njeru, and Tirimba (2014); Chittithaworn, Islam, Keawchana, and Yusuf (2011); Christina, Neelufer and Amri (2014); Business In Cameroon (2015))

Although there exists vast definitions of SMEs all across the world, Senderovitz (2009) provides a general criteria for defining SMEs in terms of the number of full time employees, annual sales turnover, and amount of total assets in the firm. Haselip, Desgain, and Mackenzie (2014) further shows some additional attributes that have been given across literature in trying to define SMEs in different countries. These include the size of the economy, the structure of its corporate sector and any relevant policy frameworks, a company's organization and behavioral characteristics, such as their degree of legal independence, small-scale decentralization, generally flatter organizational hierarchy, higher degree of informality, smaller market power and lower level of technological sophistication amongst others. In Cameroon, enterprises are classified into three categories which are, Very Small Enterprises"(VSEs), "Small Enterprises" (SEs), and "Medium-sized Enterprises"(MEs). VSE, SE and ME employ, not more than five (5) persons, between six and twenty (6-20) persons, twenty-one and no more than one hundred (21-100) persons respectively. In the same way, the respective annual turnover net of taxes for VSE, SE and ME does not exceed 15 million francs, falls between 15 and 100 million CFA francs, and lies between 100 million and 1 billion CFA francs (Afriland First Bank, 2015).

While business environments differ from economy to economy, there are some challenges that have been identified in literature as the most common hindrances to SME progress in developing countries. Bouazza, Ardjouman, and Abada (2015) separates the factors that affect the growth of SMEs into internal and those of an external nature. In their study on Algeria, the authors identified external factors such as the legal and regulatory framework, access to external financing, and human resources capacities as those of paramount importance in determining the growth of SMEs. On the internal factors side, the study identified entrepreneurial characteristics, management capacities, marketing skills, and technological capacities as the attributing factors to the unstable and limited growth of SMEs in Algeria.

In a study on the Kenyan tailoring SMEs Mbugua, Mbugua, Wangoi, Ogada, and Kariuki (2013), analyzed several owner manager characteristics as well as how these and other factors affected their enterprises. Aspects like the availability of finances, poor business management skills, poor marketing and entrepreneurial attributes of the owner managers were the main issues underlined to hamper the growth of SMEs.

\subsection{Introduction}

\section{Methodology And Procedure}

This section seeks to describe the research methodology and procedure. Sampling techniques, data collection procedure, reliability analysis, and regression analysis are discussed in detail.

\subsection{Sampling Technique and Data Collection}

While in this study particular focus is given to several factors that affect the growth and effectiveness of SMEs in developing countries, Cameroon was used as the study area. Semi structured questionnaires and face to face interviews were used to collect primary data on SMEs in Cameroon. The population consisted of 32773 Very Small, Small and Medium-sized Enterprises that were created through the Centre for the Facilitation of Creation of Enterprises (CFCE) between 2010 and 2014. According to CFCE (2015) about 52\% of these SMEs are located in Yaounde while about $42 \%$ are stationed in Douala hence the two cities were purposively selected to participate in this study.

SME data from these two cities was used as the sampling frame from which a random sample was drawn using the procedure often used across multidisciplinary studies as outlined in equation (1);

$\mathrm{n}=\frac{\mathrm{x}^{2} \mathrm{~N} \hat{\mathrm{p}}(1-\widehat{\mathrm{p}})}{\epsilon^{2}(\mathrm{~N}-1)+\mathrm{x}^{2} \widehat{\mathrm{p}}(1-\widehat{\mathrm{p}})}$

Where;

$\mathrm{n}=$ the number of respondents

$\mathrm{N}=$ population size

$\mathrm{x}^{2}=$ table value of chi-square (for 0.95 Confidence interval, $\mathrm{x}^{2}$ is given as 3.841 )

$\hat{\mathrm{p}}=$ proportion of a population that picks a choice expressed as a decimal (usually assumed to be 0.5 )

$\epsilon^{2}=$ level of error researcher is willing to tolerate (in this case $5 \%$ )

As per the criteria outlined in equation (1), the researchers utilized a sampling fraction of $2 \%$, to randomly select a total of 279 and 226 respondents from the pool of SMEs domiciled in Douala and Yaounde respectively. 
Strengthening Enterprises Growth and Effectiveness in Developing Economies. A case of Very..

\subsection{Reliability Analysis}

The reliability or internal consistency of the data set was determined by means of the Cronbach's Alpha $(\alpha)$. The chosen set of questionnaire items were all examined to assess the degree to which they measured a single unidimensional latent construct. The Cronbach's Alpha $(\alpha)$ was calculated in the form given in equation (2) below;

$\alpha=\frac{\mathrm{K}}{\mathrm{K}-1}\left(1-\frac{\sum_{\mathrm{i}=1}^{\mathrm{K}} \sigma_{\mathrm{Y}_{\mathrm{i}}}^{2}}{\sigma_{\mathrm{X}}^{2}}\right)$

Where;

$\alpha$ is the Cronbach's Alpha,

$\mathrm{K}$ is the number of components being examined,

$\sigma_{\mathrm{X}}^{2}$ is the variance of the observed total scores,

$\sigma_{\mathrm{Y}_{\mathrm{i}}}^{2}$ is the variance of component $\mathrm{i}$ for the current sample,

In line with the specification given above, internal consistency was described in accordance with the commonly accepted rule of thumb, as stated in literature, that specifies that an $\alpha$ that is less than 0.5 is unacceptable, an $\alpha$ that ranges between 0.5 and 0.6 is considered poor, an $\alpha$ that falls between 0.6 and 0.7 can be regarded as questionable, an $\alpha$ within the range of 0.7 to 0.8 is acceptable, an $\alpha$ that exceeds 0.8 but less than or equal to 0.9 is good while an $\alpha$ greater than 0.9 shows excellent internal consistency (e.g., George and Mallery (2003); DeVellis (2012)).

\subsection{Pearson's Correlation}

The factors that have an impact on the SME growth and effectiveness were empirically analyzed by means of the Pearson's Correlation coefficient. This method helped determine the strength and direction of all linear relationships between the paired data. The criterion was computed as given in equation (3);

$r=\frac{\sum_{i=1}^{n}\left(x_{i}-\bar{x}\right)\left(y_{i}-\bar{y}\right)}{\sqrt{\sum_{i=1}^{n}\left(x_{i}-\bar{x}\right)^{2}} \sqrt{\sum_{i=1}^{n}\left(y_{i}-\bar{y}\right)^{2}}}$

Where;

$r$ is the Pearson's correlation coefficient for the sample,

$\mathrm{n}$ is the sample size,

$\mathrm{x}_{\mathrm{i}}$ refers to the terms in the dataset $\left\{\mathrm{x}_{1}, \ldots, \mathrm{x}_{\mathrm{n}}\right\}$,

$\overline{\mathrm{x}}$ is the sample mean calculated as $\frac{1}{\mathrm{n}} \sum_{\mathrm{i}=1}^{\mathrm{n}} \mathrm{x}_{\mathrm{i}}$,

$\mathrm{y}_{\mathrm{i}}$ refers to the terms in the dataset $\left\{\mathrm{y}_{1}, \ldots, \mathrm{y}_{\mathrm{n}}\right\}$,

$\overline{\mathrm{y}}$ is the sample mean calculated as $\frac{1}{\mathrm{n}} \sum_{\mathrm{i}=1}^{\mathrm{n}} \mathrm{y}_{\mathrm{i}}$.

In line with the specification in equation 3, the Pearson's correlation coefficient ( $r$ ) was interpreted in accordance with the commonly accepted rule of thumb. Lund and Lund (2013) specifies that the strength of association is considered small when $\mathrm{r}$ is between 0.1 and 0.3 . The authors further expound that the association is medium and large when the magnitude of $\mathrm{r}$ falls between 0.3 and 0.5 as well as between 0.5 and 1.0 respectively as summarized in Table 1 .

Table 1: Decision rule of thumb regarding Pearson's correlation coefficient (r)

\begin{tabular}{|l|l|l|}
\hline & \multicolumn{2}{|c|}{ Coefficient, $\mathrm{r}$} \\
\hline Strength of Association & Positive & Negative \\
\hline Small & 0.1 to 0.3 & -0.1 to -0.3 \\
\hline Medium & 0.3 to 0.5 & -0.3 to -0.5 \\
\hline Large & 0.5 to 1.0 & -0.5 to -1.0 \\
\hline
\end{tabular}

Source: (Lund and Lund, 2013)

\section{Results Of Study}

This study achieved a response rate of $79 \%$ hence the results presented hereunder is based on the analysis of data collected from 400 duly completed questionnaires.

\subsection{Reasons for starting business}

While a business' success is a function of many internal and external environmental factors, it can be argued that an entrepreneur's motive to start the business has a role to play in the survival and growth of their enterprises. $21 \%$ of the respondents ventured into business as a last resort. These are the entrepreneurs who opened companies as a desperation move after all other avenues seemed to have closed for them. About 33\% only thought of opening some enterprises as a survival strategy when they had lost their previous jobs. Some 
other respondents indicated that they began their small companies only to raise an extra income to supplement the salaries from their main jobs. This group of entrepreneurs runs business on a part time basis. There is also a significant number of respondents who indicated that they are deeply immersed in business and they opened their SMEs as a matter of priority to fulfill their inborn desire to do business. This is the group of entrepreneurs who operate on a fulltime basis and results indicate that they are amongst those who have registered good growth and have also created more employment over time.

\subsection{Business Management related training}

The study found out that the majority of the respondents have at least basic knowledge and or training in the functional areas of business. For instance, about $51 \%$ of the respondents are knowledgeable with financial management, 58\% with human resource management, $66 \%$ with business administration, and $71 \%$ with marketing management. The proportion of those deficient in these skills remain significant and that creates an opportunity to roll out general management training courses to boost up the quality of SME managers in Cameroon.

\subsection{Scale reliability}

Table 2 shows the Cronbach's alpha as a measure of internal consistency. The subsequent discussion presents the variables that were found to be closely related as a group according to the Cronbach's alpha reliability tool.

Table 2: Reliability Statistics

\begin{tabular}{|l|l|}
\hline Cronbach's Alpha & $\mathrm{N}$ of Items \\
\hline 0.700 & 18 \\
\hline
\end{tabular}

The Cronbach's alpha (0.700) shows that 18 of the survey instrument's items satisfactorily measured a single unidimensional latent construct. These items that were found to be statistically reliable include SME growth and effectiveness, entrepreneur's level of education, entrepreneur's experience, owner management of SMEs, the relevance of entrepreneur's qualifications, their financial management skills, budgeting ability, human resources management skills, administration skills, operations management skills, research and development ability, marketing management skills, provision of product education, incidence of corruption the business environment, availability of a business bank account, propensity to own land, level of competition on the market, as well as the level of SMEs confidence in the economic system.

\subsection{Factors affecting SMEs' effectiveness}

Table 3 presents the linear relationships between variables that were found to significantly have an impact on SMEs growth and effectiveness. The sign of each coefficient in Table 3 represents the direction of the linear correlation while their magnitudes show the strength of each linear relationship between pertinent variables in question.

Table 3: Linear relationships between variables that impact on SMEs growth and effectiveness

\begin{tabular}{|c|c|c|c|c|c|c|c|c|c|c|c|c|c|c|c|c|c|c|c|}
\hline & A & B & C & $\mathrm{D}$ & E & F & $\mathrm{G}$ & $\mathrm{H}$ & I & $\mathbf{J}$ & K & L & $\mathrm{M}$ & $\mathrm{N}$ & $\mathrm{O}$ & $\mathrm{P}$ & $\mathrm{Q}$ & $\mathrm{R}$ & S \\
\hline $\mathrm{A}$ & 1 & $\begin{array}{l}.38 \\
6^{* * *}\end{array}$ & $\begin{array}{l}.50 \\
2^{* * *}\end{array}$ & $\begin{array}{l}.36 \\
4^{*} \\
*\end{array}$ & $\begin{array}{l}.20 \\
3^{*}\end{array}$ & $\begin{array}{l}.59 \\
2^{* * *}\end{array}$ & $\begin{array}{l}.22 \\
3^{*}\end{array}$ & $\begin{array}{l}.38 \\
0^{* *}\end{array}$ & $\begin{array}{l}.30 \\
7^{* *}\end{array}$ & $\begin{array}{l}.45 \\
3^{* *}\end{array}$ & $\begin{array}{l}.40 \\
9^{* *}\end{array}$ & $\begin{array}{l}.33 \\
6 * *\end{array}$ & $\begin{array}{l}.20 \\
6^{*}\end{array}$ & $\begin{array}{l}- \\
.67 \\
7 * *\end{array}$ & $\begin{array}{l}.23 \\
0^{*}\end{array}$ & $\begin{array}{l}.23 \\
5^{*}\end{array}$ & $\begin{array}{l}.24 \\
1^{*}\end{array}$ & $\begin{array}{l}- \\
.21 \\
9^{*}\end{array}$ & $\begin{array}{l}.67 \\
2 * *\end{array}$ \\
\hline B & $\begin{array}{l}.38 \\
6^{* *}\end{array}$ & 1 & $\begin{array}{l}.20 \\
7^{*}\end{array}$ & $\begin{array}{l}0.1 \\
3\end{array}$ & $\begin{array}{l}0.0 \\
4\end{array}$ & $\begin{array}{l}.34 \\
9 * *\end{array}$ & $\begin{array}{l}.21 \\
7^{*}\end{array}$ & $\begin{array}{l}0.1 \\
8\end{array}$ & $\begin{array}{l}0.0 \\
8\end{array}$ & $\begin{array}{l}0.1 \\
4\end{array}$ & $\begin{array}{l}.21 \\
8^{*}\end{array}$ & 0.1 & $\begin{array}{l}0.1 \\
2\end{array}$ & $\begin{array}{l}- \\
.23 \\
2^{*}\end{array}$ & $\begin{array}{l}- \\
0.0 \\
5\end{array}$ & $\begin{array}{l}- \\
0.0 \\
7\end{array}$ & $\begin{array}{l}0.0 \\
4\end{array}$ & $\begin{array}{l}- \\
.24 \\
7 *\end{array}$ & $\begin{array}{l}.32 \\
0 * *\end{array}$ \\
\hline $\mathrm{C}$ & $\begin{array}{l}.50 \\
2^{* *}\end{array}$ & $\begin{array}{l}.20 \\
7^{*}\end{array}$ & 1 & $\begin{array}{l}.21 \\
7^{*}\end{array}$ & $\begin{array}{l}0.0 \\
1\end{array}$ & $\begin{array}{l}.28 \\
2 * *\end{array}$ & $\begin{array}{l}0.1 \\
2\end{array}$ & $\begin{array}{l}.26 \\
7 * *\end{array}$ & 0.1 & $\begin{array}{l}0.1 \\
9\end{array}$ & $\begin{array}{l}0.0 \\
7\end{array}$ & $\begin{array}{l}0.1 \\
4\end{array}$ & $\begin{array}{l}0.0 \\
9\end{array}$ & $\begin{array}{l}- \\
.40 \\
0 * *\end{array}$ & $\begin{array}{l}- \\
0.0 \\
2\end{array}$ & $\begin{array}{l}.20 \\
5^{*}\end{array}$ & $\begin{array}{l}0.1 \\
2\end{array}$ & $\begin{array}{l}- \\
0.1 \\
6\end{array}$ & $\begin{array}{l}.39 \\
6^{* *}\end{array}$ \\
\hline $\mathrm{D}$ & $\begin{array}{l}.36 \\
4 * *\end{array}$ & $\begin{array}{l}0.1 \\
3\end{array}$ & $\begin{array}{l}.21 \\
7^{*}\end{array}$ & 1 & $\begin{array}{l}0.1 \\
3\end{array}$ & $\begin{array}{l}- \\
0.0 \\
7\end{array}$ & $\begin{array}{l}0.0 \\
9\end{array}$ & $\begin{array}{l}0.1 \\
2\end{array}$ & $\begin{array}{l}.20 \\
9^{*}\end{array}$ & $\begin{array}{l}0.1 \\
2\end{array}$ & $\begin{array}{l}0.0 \\
7\end{array}$ & $\begin{array}{l}0.1 \\
2\end{array}$ & 0.1 & $\begin{array}{l}- \\
.26 \\
5 * *\end{array}$ & $\begin{array}{l}.19 \\
7^{*}\end{array}$ & $\begin{array}{l}0.0 \\
8\end{array}$ & $\begin{array}{l}0.1 \\
2\end{array}$ & $\begin{array}{l}- \\
0.0 \\
1\end{array}$ & $\begin{array}{l}.23 \\
5^{*}\end{array}$ \\
\hline $\mathrm{E}$ & $\begin{array}{l}.20 \\
3^{*}\end{array}$ & $\begin{array}{l}0.0 \\
4\end{array}$ & $\begin{array}{l}0.0 \\
1\end{array}$ & $\begin{array}{l}0.1 \\
3\end{array}$ & 1 & $\begin{array}{l}0.1 \\
4\end{array}$ & 0 & $\begin{array}{l}0.0 \\
4\end{array}$ & $\begin{array}{l}- \\
0.0 \\
8\end{array}$ & $\begin{array}{l}.34 \\
1^{* *}\end{array}$ & $\begin{array}{l}.25 \\
7^{* *}\end{array}$ & $\begin{array}{l}.24 \\
2^{*}\end{array}$ & 0 & -0.1 & $\begin{array}{l}- \\
0.0 \\
8\end{array}$ & $\begin{array}{l}0.1 \\
8\end{array}$ & $\begin{array}{l}0.0 \\
7\end{array}$ & $\begin{array}{l}- \\
0.0 \\
8\end{array}$ & 0.1 \\
\hline $\mathrm{F}$ & $\begin{array}{l}.59 \\
2^{* * *}\end{array}$ & $\begin{array}{l}.34 \\
9^{* *}\end{array}$ & $\begin{array}{l}.28 \\
2^{* *}\end{array}$ & $\begin{array}{l}- \\
0.0 \\
7 \\
\end{array}$ & $\begin{array}{l}0.1 \\
4\end{array}$ & 1 & $\begin{array}{l}0.1 \\
5\end{array}$ & $\begin{array}{l}.30 \\
1 * *\end{array}$ & $\begin{array}{l}0.1 \\
4\end{array}$ & $\begin{array}{l}.36 \\
2 * *\end{array}$ & $\begin{array}{l}.48 \\
5 * *\end{array}$ & $\begin{array}{l}0.0 \\
4\end{array}$ & $\begin{array}{l}.21 \\
0^{*}\end{array}$ & $\begin{array}{l}- \\
.48 \\
0 * *\end{array}$ & $\begin{array}{l}0.0 \\
6\end{array}$ & $\begin{array}{l}- \\
0.0 \\
3 \\
\end{array}$ & $\begin{array}{l}0.1 \\
7\end{array}$ & $\begin{array}{l}- \\
0.0 \\
6 \\
\end{array}$ & $\begin{array}{l}.36 \\
1 * *\end{array}$ \\
\hline $\mathrm{G}$ & $\begin{array}{l}.22 \\
3^{*}\end{array}$ & $\begin{array}{l}.21 \\
7^{*}\end{array}$ & $\begin{array}{l}0.1 \\
2\end{array}$ & $\begin{array}{l}0.0 \\
9\end{array}$ & 0 & $\begin{array}{l}0.1 \\
5\end{array}$ & 1 & $\begin{array}{l}0.0 \\
1\end{array}$ & $\begin{array}{l}.21 \\
1^{*}\end{array}$ & $\begin{array}{l}0.0 \\
5\end{array}$ & $\begin{array}{l}0.1 \\
9\end{array}$ & $\begin{array}{l}0.0 \\
4\end{array}$ & $\begin{array}{l}0.0 \\
4\end{array}$ & $\begin{array}{l}0.0 \\
1 \\
\end{array}$ & $\begin{array}{l}.24 \\
8^{*}\end{array}$ & $\begin{array}{l}0.0 \\
4\end{array}$ & $\begin{array}{l}- \\
.20 \\
2^{*}\end{array}$ & $\begin{array}{l}0.0 \\
6 \\
\end{array}$ & $\begin{array}{l}0.1 \\
7\end{array}$ \\
\hline $\mathrm{H}$ & $\begin{array}{l}.38 \\
0^{* *}\end{array}$ & $\begin{array}{l}0.1 \\
8\end{array}$ & $\begin{array}{l}.26 \\
7^{* *}\end{array}$ & $\begin{array}{l}0.1 \\
2\end{array}$ & $\begin{array}{l}0.0 \\
4\end{array}$ & $\begin{array}{l}.30 \\
1 * *\end{array}$ & $\begin{array}{l}0.0 \\
1\end{array}$ & 1 & $\begin{array}{l}.45 \\
9^{* *}\end{array}$ & $\begin{array}{l}.23 \\
3^{*}\end{array}$ & $\begin{array}{l}0.1 \\
1\end{array}$ & $\begin{array}{l}0.1 \\
3\end{array}$ & $\begin{array}{l}.26 \\
0^{* * *}\end{array}$ & $\begin{array}{l}- \\
.38 \\
2 * *\end{array}$ & $\begin{array}{l}.35 \\
9^{* *}\end{array}$ & 0 & $\begin{array}{l}0.0 \\
4\end{array}$ & $\begin{array}{l}- \\
.20 \\
9^{*}\end{array}$ & $\begin{array}{l}.30 \\
7 * *\end{array}$ \\
\hline
\end{tabular}


Strengthening Enterprises Growth and Effectiveness in Developing Economies. A case of Very..

\begin{tabular}{|c|c|c|c|c|c|c|c|c|c|c|c|c|c|c|c|c|c|c|c|}
\hline I & $\begin{array}{l}.30 \\
7 * *\end{array}$ & $\begin{array}{l}0.0 \\
8\end{array}$ & 0.1 & $\begin{array}{l}.20 \\
9^{*}\end{array}$ & $\begin{array}{l}- \\
0.0 \\
8 \\
\end{array}$ & $\begin{array}{l}0.1 \\
4\end{array}$ & $\begin{array}{l}.21 \\
1^{*}\end{array}$ & $\begin{array}{l}.45 \\
9 * *\end{array}$ & 1 & $\begin{array}{l}.29 \\
5 * *\end{array}$ & $\begin{array}{l}0.1 \\
9\end{array}$ & $\begin{array}{l}0.0 \\
5\end{array}$ & $\begin{array}{l}0.1 \\
2\end{array}$ & $\begin{array}{l}- \\
.31 \\
0 * *\end{array}$ & $\begin{array}{l}.36 \\
7^{* * *}\end{array}$ & $\begin{array}{l}0.0 \\
1\end{array}$ & $\begin{array}{l}- \\
0.0 \\
9 \\
\end{array}$ & $\begin{array}{l}- \\
0.0 \\
6\end{array}$ & $\begin{array}{l}.32 \\
3^{* *}\end{array}$ \\
\hline $\mathbf{J}$ & $\begin{array}{l}.45 \\
3^{* *}\end{array}$ & $\begin{array}{l}0.1 \\
4\end{array}$ & $\begin{array}{l}0.1 \\
9\end{array}$ & $\begin{array}{l}0.1 \\
2\end{array}$ & $\begin{array}{l}.34 \\
1 * \\
*\end{array}$ & $\begin{array}{l}.36 \\
2^{* *} \\
\end{array}$ & $\begin{array}{l}0.0 \\
5 \\
\end{array}$ & $\begin{array}{l}.23 \\
3^{*}\end{array}$ & $\begin{array}{l}.29 \\
5 * *\end{array}$ & 1 & $\begin{array}{l}.21 \\
6^{*} \\
\end{array}$ & $\begin{array}{l}.33 \\
7 * *\end{array}$ & $\begin{array}{l}0.0 \\
4 \\
\end{array}$ & $\begin{array}{l}- \\
.24 \\
2^{*}\end{array}$ & $\begin{array}{l}0.1 \\
8\end{array}$ & $\begin{array}{l}0.0 \\
6 \\
\end{array}$ & $\begin{array}{l}0.1 \\
6\end{array}$ & $\begin{array}{l}-0.1 \\
8 \\
\end{array}$ & $\begin{array}{l}.31 \\
9 * *\end{array}$ \\
\hline $\mathrm{K}$ & $\begin{array}{l}.40 \\
9 * *\end{array}$ & $\begin{array}{l}.21 \\
8^{*}\end{array}$ & $\begin{array}{l}0.0 \\
7\end{array}$ & $\begin{array}{l}0.0 \\
7\end{array}$ & $\begin{array}{l}.25 \\
7 * \\
*\end{array}$ & $\begin{array}{l}.48 \\
5 * *\end{array}$ & $\begin{array}{l}0.1 \\
9\end{array}$ & $\begin{array}{l}0.1 \\
1\end{array}$ & $\begin{array}{l}0.1 \\
9\end{array}$ & $\begin{array}{l}.21 \\
6^{*}\end{array}$ & 1 & $\begin{array}{l}0.1 \\
3\end{array}$ & $\begin{array}{l}0.0 \\
5\end{array}$ & $\begin{array}{l}- \\
.27 \\
1 * *\end{array}$ & $\begin{array}{l}0.0 \\
3\end{array}$ & $\begin{array}{l}- \\
0.0 \\
2\end{array}$ & $\begin{array}{l}0.0 \\
3\end{array}$ & $\begin{array}{l}- \\
0.1 \\
9\end{array}$ & $\begin{array}{l}.28 \\
6^{* * *}\end{array}$ \\
\hline $\mathrm{L}$ & $\begin{array}{l}.33 \\
6^{* *}\end{array}$ & 0.1 & $\begin{array}{l}0.1 \\
4\end{array}$ & $\begin{array}{l}0.1 \\
2\end{array}$ & $\begin{array}{l}.24 \\
2^{*}\end{array}$ & $\begin{array}{l}0.0 \\
4\end{array}$ & $\begin{array}{l}0.0 \\
4\end{array}$ & $\begin{array}{l}0.1 \\
3\end{array}$ & $\begin{array}{l}0.0 \\
5\end{array}$ & $\begin{array}{l}.33 \\
7 * *\end{array}$ & $\begin{array}{l}0.1 \\
3\end{array}$ & 1 & $\begin{array}{l}- \\
0.0 \\
4\end{array}$ & $\begin{array}{l}- \\
.25 \\
5 * \\
\end{array}$ & $\begin{array}{l}0.1 \\
4\end{array}$ & $\begin{array}{l}0.0 \\
2\end{array}$ & $\begin{array}{l}0.1 \\
3\end{array}$ & $\begin{array}{l}- \\
.26 \\
1 * * \\
\end{array}$ & $\begin{array}{l}.23 \\
0^{*}\end{array}$ \\
\hline $\mathrm{M}$ & $\begin{array}{l}.20 \\
6^{*}\end{array}$ & $\begin{array}{l}0.1 \\
2\end{array}$ & $\begin{array}{l}0.0 \\
9\end{array}$ & 0.1 & 0 & $\begin{array}{l}.21 \\
0^{*}\end{array}$ & $\begin{array}{l}0.0 \\
4\end{array}$ & $\begin{array}{l}.26 \\
0 * *\end{array}$ & $\begin{array}{l}0.1 \\
2\end{array}$ & $\begin{array}{l}0.0 \\
4\end{array}$ & $\begin{array}{l}0.0 \\
5\end{array}$ & $\begin{array}{l}- \\
0.0 \\
4\end{array}$ & 1 & $\begin{array}{l}- \\
.21 \\
0^{*}\end{array}$ & $\begin{array}{l}0.0 \\
6\end{array}$ & $\begin{array}{l}0.1 \\
2\end{array}$ & $\begin{array}{l}.30 \\
0^{* *}\end{array}$ & $\begin{array}{l}.19 \\
8^{*}\end{array}$ & $\begin{array}{l}0.0 \\
6\end{array}$ \\
\hline $\mathrm{N}$ & $\begin{array}{l}- \\
.67 \\
7^{* *}\end{array}$ & $\begin{array}{l}- \\
.23 \\
2^{*}\end{array}$ & $\begin{array}{l}- \\
.40 \\
0^{* *}\end{array}$ & $\begin{array}{l}- \\
.26 \\
5^{*} \\
*\end{array}$ & $\overline{0} .1$ & $\begin{array}{l}- \\
.48 \\
0 * *\end{array}$ & $\begin{array}{l}- \\
0.0 \\
1\end{array}$ & $\begin{array}{l}- \\
.38 \\
2 * *\end{array}$ & $\begin{array}{l}- \\
.31 \\
0^{* *}\end{array}$ & $\begin{array}{l}- \\
.24 \\
2^{*}\end{array}$ & $\begin{array}{l}- \\
.27 \\
1 * *\end{array}$ & $\begin{array}{l}- \\
.25 \\
5^{*}\end{array}$ & $\begin{array}{l}- \\
.21 \\
0^{*}\end{array}$ & 1 & -0.1 & $\begin{array}{l}- \\
0.1 \\
5\end{array}$ & $\begin{array}{l}- \\
0.1 \\
2\end{array}$ & $\begin{array}{l}0.0 \\
6\end{array}$ & $\begin{array}{l}- \\
.60 \\
1 * *\end{array}$ \\
\hline $\mathrm{O}$ & $\begin{array}{l}.23 \\
0^{*}\end{array}$ & $\begin{array}{l}- \\
0.0 \\
5 \\
\end{array}$ & $\begin{array}{l}- \\
0.0 \\
2 \\
\end{array}$ & $\begin{array}{l}.19 \\
7^{*}\end{array}$ & $\begin{array}{l}- \\
0.0 \\
8 \\
\end{array}$ & $\begin{array}{l}0.0 \\
6\end{array}$ & $\begin{array}{l}.24 \\
8^{*}\end{array}$ & $\begin{array}{l}.35 \\
9 * *\end{array}$ & $\begin{array}{l}.36 \\
7 * *\end{array}$ & $\begin{array}{l}0.1 \\
8\end{array}$ & $\begin{array}{l}0.0 \\
3\end{array}$ & $\begin{array}{l}0.1 \\
4\end{array}$ & $\begin{array}{l}0.0 \\
6\end{array}$ & -0.1 & 1 & $\begin{array}{l}- \\
0.0 \\
2 \\
\end{array}$ & $\begin{array}{l}- \\
0.1 \\
7 \\
\end{array}$ & $\begin{array}{l}- \\
0.0 \\
4 \\
\end{array}$ & 0.1 \\
\hline $\mathrm{P}$ & $\begin{array}{l}.23 \\
5^{*}\end{array}$ & $\begin{array}{l}- \\
0.0 \\
7 \\
\end{array}$ & $\begin{array}{l}.20 \\
5^{*}\end{array}$ & $\begin{array}{l}0.0 \\
8\end{array}$ & $\begin{array}{l}0.1 \\
8\end{array}$ & $\begin{array}{l} \\
0.0 \\
3 \\
\end{array}$ & $\begin{array}{l}0.0 \\
4\end{array}$ & 0 & $\begin{array}{l}0.0 \\
1\end{array}$ & $\begin{array}{l}0.0 \\
6\end{array}$ & $\begin{array}{l}- \\
0.0 \\
2 \\
\end{array}$ & $\begin{array}{l}0.0 \\
2\end{array}$ & $\begin{array}{l}0.1 \\
2\end{array}$ & $\begin{array}{l}- \\
0.1 \\
5 \\
\end{array}$ & $\begin{array}{l}- \\
0.0 \\
2 \\
\end{array}$ & 1 & $\begin{array}{l}- \\
0.0 \\
3 \\
\end{array}$ & $\begin{array}{l}0.0 \\
2\end{array}$ & $\begin{array}{l}0.1 \\
7\end{array}$ \\
\hline $\mathrm{Q}$ & $\begin{array}{l}.24 \\
1^{*}\end{array}$ & $\begin{array}{l}0.0 \\
4\end{array}$ & $\begin{array}{l}0.1 \\
2\end{array}$ & $\begin{array}{l}0.1 \\
2\end{array}$ & $\begin{array}{l}0.0 \\
7\end{array}$ & $\begin{array}{l}0.1 \\
7\end{array}$ & $\begin{array}{l}- \\
.20 \\
2 *\end{array}$ & $\begin{array}{l}0.0 \\
4\end{array}$ & $\begin{array}{l}- \\
0.0 \\
9\end{array}$ & $\begin{array}{l}0.1 \\
6\end{array}$ & $\begin{array}{l}0.0 \\
3\end{array}$ & $\begin{array}{l}0.1 \\
3\end{array}$ & $\begin{array}{l}.30 \\
0 * *\end{array}$ & $\begin{array}{l}- \\
0.1 \\
2 \\
\end{array}$ & $\begin{array}{l}- \\
0.1 \\
7 \\
\end{array}$ & $\begin{array}{l}- \\
0.0 \\
3 \\
\end{array}$ & 1 & 0 & $\begin{array}{l}0.0 \\
5\end{array}$ \\
\hline $\mathrm{R}$ & $\begin{array}{l}- \\
.21 \\
9^{*} \\
\end{array}$ & $\begin{array}{l}- \\
.24 \\
7 * \\
\end{array}$ & $\begin{array}{l}- \\
0.1 \\
6 \\
\end{array}$ & $\begin{array}{l}- \\
0.0 \\
1 \\
\end{array}$ & $\begin{array}{l}- \\
0.0 \\
8 \\
\end{array}$ & $\begin{array}{l}- \\
0.0 \\
6 \\
\end{array}$ & $\begin{array}{l}- \\
0.0 \\
6 \\
\end{array}$ & $\begin{array}{l}- \\
.20 \\
9 * \\
\end{array}$ & $\begin{array}{l}- \\
0.0 \\
6 \\
\end{array}$ & $\begin{array}{l}- \\
0.1 \\
8 \\
\end{array}$ & $\begin{array}{l}- \\
0.1 \\
9 \\
\end{array}$ & $\begin{array}{l}- \\
.26 \\
1 * *\end{array}$ & $\begin{array}{l}.19 \\
8^{*}\end{array}$ & $\begin{array}{l}0.0 \\
6\end{array}$ & $\begin{array}{l}- \\
0.0 \\
4 \\
\end{array}$ & $\begin{array}{l}0.0 \\
2\end{array}$ & 0 & 1 & $\begin{array}{l}- \\
.29 \\
9 * * \\
\end{array}$ \\
\hline $\mathrm{S}$ & $\begin{array}{l}.67 \\
2 * *\end{array}$ & $\begin{array}{l}.32 \\
0^{* *}\end{array}$ & $\begin{array}{l}.39 \\
6^{* *}\end{array}$ & $\begin{array}{l}.23 \\
5^{*}\end{array}$ & 0.1 & $\begin{array}{l}.36 \\
1 * *\end{array}$ & $\begin{array}{l}0.1 \\
7\end{array}$ & $\begin{array}{l}.30 \\
7 * *\end{array}$ & $\begin{array}{l}.32 \\
3^{* *}\end{array}$ & $\begin{array}{l}.31 \\
9 * *\end{array}$ & $\begin{array}{l}.28 \\
6^{* *}\end{array}$ & $\begin{array}{l}.23 \\
0^{*}\end{array}$ & $\begin{array}{l}0.0 \\
6\end{array}$ & $\begin{array}{l}- \\
.60 \\
1 * *\end{array}$ & 0.1 & $\begin{array}{l}0.1 \\
7\end{array}$ & $\begin{array}{l}0.0 \\
5\end{array}$ & $\begin{array}{l}- \\
.29 \\
9 * *\end{array}$ & 1 \\
\hline
\end{tabular}

** Correlation is significant at the 0.01 level (2-tailed).

* Correlation is significant at the 0.05 level (2-tailed).

According to the Pearson's Correlation coefficient matrix in Table 3, about 18 variables were found to be important in explaining the SME growth and effectiveness in Cameroon. In Table 3 each of these variables is denoted by a capital letter and each subsequent letter is defined as follows;

A- SME growth and effectiveness, B - Entrepreneur's level of Education, C- Entrepreneur's Experience, D- Owner management, E- Relevance of entrepreneur's qualification, F- Financial management skills, G -Budgeting ability, H- Human resources management skills, I - Administration Skills, J- Operations Management skills, K- Research and Development ability, L - Marketing Management skills, M- Provision of Product Education, N- Corruption, O- availability of a business bank account, P- Propensity to own land, Q Competition, $\mathrm{R}$ - Crime and Theft, $\mathrm{S}$ - Confidence in the economic system.

SME growth and effectiveness as demonstrated by the entrepreneur's ability to create more and more employment was the dependent variable while the rest other variables were explanatory. Table 3 shows that $22 \%$ of the variables display strong linear correlations with SME growth and effectiveness. On the other hand, $39 \%$ and $38.9 \%$ of the explanatory variables in Table 3 respectively exhibit moderate and weak correlations with the SME growth and effectiveness. $61 \%$ of the correlations in Table 3 were significant at 0.01 level while the rest were significant at 0.05 level for a two tailed test.

The entrepreneur's level of experience, their financial management skills, as well as their confidence in the political and economic future of the country all displayed the strongest correlation with the SMEs growth and effectiveness. Also showing a strong correlation with the dependent variable is the level of corruption in the economy. The entrepreneur's level of experience significantly has a positive impact $(r=0.502)$ on the SME's ability to grow. SME's who have been in operation for longer periods of time also demonstrated a remarkable ability to employ more workforce effectively. On the contrary lesser job creation ability was recorded from relatively new SME's. It is therefore recommended that the government through responsible bodies introduce and or foster mentorship programs to cater for new and upcoming SMEs. Working under an experienced expert in their respective fields helps them overcome a lot of challenges that often humper the progress of start-ups. Since experience is a good teacher, experienced mentors usually know what to expect and how to react to the industry or market dynamics. Once they are experienced enough, trainee SMEs can then be released to operate independently and according to the results of this study they can by this time perform more optimally and effectively. 
Possessing financial management skills proved to be an important prerequisite of SME success. The study found a strong and positive correlation $(\mathrm{r}=0.592)$ between financial management skills and SME growth and effectiveness. Those enterprises that are run by financially literate personnel registered better performance than their counterparts whose staff did not undergo any financial management training. The less financially qualified SME operators were found to have a lesser chance to grow and develop effectively. Since according to this study, financial management skills are a crucial trait of most successful SMEs, it is difficult to make it in business without the necessary financial abilities. It therefore becomes imperative to roll-out nation-wide training programs to educate SME operators on subjects like financial literacy, basic accounting and book keeping, financial statements and so on.

The degree of business confidence in the economy was also found to be an important determinant of SME growth in Cameroon. With a strong and positive Pearson's correlation coefficient of 0.672 , it can be deduced that SMEs who are confident in the country's political situation as well as the economic growth trajectory are likely to perform better than those that are doubtful. Apprehensive SME owners in many cases find it difficult to take calculated risky decisions and investments which when done may actually bring forth good prospects for their businesses. It was also found in this study that some SMEs who are uncertain about the economic future of the country usually prefer to operate on a small scale for the fear of losing control of their business. It is crucial for politicians and policy makers to cultivate the spirit of optimism amongst citizens through the way they run the economy. The government by means of its monetary and fiscal policies should ensure that there is continually favorable employment levels, high consumer confidence, low inflation and interest rates in the economy so as to safeguard SME confidence in turn.

The prevalence of corruption in the Cameroonian business setup registered a great likelihood to slow down the growth of SMEs. Table 3 shows that corruption as an explanatory variable has a strong and inverse Pearson's correlation coefficient of -0.677 with the dependent variable. SMEs who have been victims of this economic rot found it very difficult to grow effectively. Some SMEs allegedly kept paying authorities for services that should have been provided to them at no charge and this has proven to eat into their hard earned profits. On the other hand, SMEs who have been adamant on their position against corruption did not pay any bribes but were reportedly given last preference in getting their things done in public offices. As a result, corruption has shown some evidence of slowing SME performance whether the operators engage in or do not participate in corrupt activities. According to a corruption report by GAN Integrity Inc. (2015), the costs and risks of doing business in Cameroon are high due to the endemic nature of corruption in the country. This report states that bribery, nepotism and corruption are rife in Cameroon's judicial system, customs and the public services. It is therefore strongly recommended that the government of Cameroon toughen as well as intensify the crackdown on corruption in the country to improve the ease of doing business in the country.

As shown in Table 3 variables such as the entrepreneur's level of education, SME owner management, entrepreneur's human resources management skills, their administration skills, operations management skills, research and development ability and marketing management skills, exhibited moderate correlation with SME growth and effectiveness. All of these correlations were unidirectional and positive.

This study found that the entrepreneur's level of education has $(r=0.386)$ a moderate and positive linear relationship with SME's ability to grow effectively. The more years an entrepreneur invests in acquiring academic and professional knowledge, the greater is their likelihood to lead successful business ventures. SMEs that were managed by educated personnel registered more growth than those whose superiors are less educated. Less educated entrepreneurs in most cases find it difficult to apply economic theory in solving their day to day business challenges. Educated entrepreneurs usually thrive in their logical reasoning capabilities, responsiveness and adaptability as well as in the application of business principles and techniques and all these go a long way in helping them grow their businesses effectively. This finding reinforces the fact that education remains a paramount cornerstone to the development of any economy. With this in mind, more opportunities should therefore be created for entrepreneurs to further their formal or informal education through vocational training, scholarships, grants, college bursaries, fellowships, and so on. The government may also offer incentives such as tax reliefs for entrepreneurs who possess certain academic qualifications as a way to encourage studying amongst SME operators.

A positive and moderately strong correlation $(r=0.364)$ was also found to exist between SME growth and effectiveness and owner management. Owners of SMEs who were actively involved in the day to day running of their own businesses demonstrated more growth and effectiveness than those who are dormant business managers. This study established that owner managers were usually more flexible to make quick and sometimes sudden decisions especially where necessary adjustments were essential to meet the ever dynamic business conditions. Moreover, owners who manage their own SMEs were mostly motivated by maximizing their wealth growth and they would deliver exceptional and more personal customer service hence registering more business growth and effective customer service. Efforts by the government should therefore be focused on producing a caliber of academic graduates who are entrepreneurial and innovative enough to open their own 
companies after studies. By so doing Cameroon will have more job creators that offset population of job seekers and ultimately, most SMEs will be managed by their owners.

While a number of functional areas of business are important in ensuring business success and prosperity, human resources management skills were found to be an important ingredient for SME growth and effectiveness. The variable for this functional area of business exhibited a positive and moderately strong correlation $(r=0.380)$ with SME growth. SME operators who at some point underwent some training in human resource management achieved more growth in terms of job creation than their non-trained counterparts. This is because they are better capacitated to maximize employee performance and this in turn helps them achieve the SME's strategic objectives. Some of the practices that the researchers identified on successful Cameroonian SMEs include; the presence of a recruitment plan, continual on-job training and development, financial and nonfinancial employee incentives, employees performance appraisals and recognition of achievements amongst others. It is therefore recommended that the government must launch a workshop program for all SME operators to train them on important human resources management issues.

Administration skills also proved to have a significant positive influence on the SMEs growth and effectiveness. A Pearson's correlation coefficient of 0.307 indicates that administration skills have a moderately strong impact on an SME's ability to prosper. In this study, most of the SMEs that displayed growth also put value on the efficient planning, organization, and control of people and other resources. Entrepreneurs with administration-related training showed more growth as compared to those who did not possess this crucial skill. Moreover, SMEs with success stories are managed with individuals who are computer literate and make use of management information systems to maintain files of important business records. SME growth and effectiveness is dependent upon the proper management of business operations, people, as well as other resources in order to meet the goals and objectives of the business entity. SME growth and effectiveness is also reliant upon the intelligent decision making ability of managers which the government can warrant by unleashing long distance programs that offer scholarships to entrepreneurs who wish to study business administration at certificate, bachelors, masters or doctorate level.

The variable for Operations Management skills was found to significantly correlate with the dependent variable. It is not surprising why the correlation coefficient 0.453 indicates a moderately strong positive relationship between the possibilities of an SME growing and whether the entrepreneur or the manager has operation management skills. According to this study, SMEs that operated optimally had a competent manager or owner who from time to time seeks to minimize costs in the production process as a way to maximize revenue for the SME. Most of the successful Cameroonian SMEs plan before they begin with the production process. They also have well managed storage facilities for their products and materials. This study also found out that the majority of successful SMEs take stock of their materials, semi- finished products and the completed goods from time to time. These SMEs are the same ones who also keep record of production and sales history for future planning. The importance of operations management skills in an SME set up cannot be under estimated. Business units usually thrive when they are led by individuals who are good at purchasing and supply management, quality management, storage and inventory control, logistics and other important roles concerned with the overseeing, designing, and controlling of the production and/or business processes.

The study also found out that Cameroonian SMEs who continually research on new production methods to improve their product quality usually grow over time and they are also effective in meeting their client's needs. Empirical evidence is given in Table 3 where the Pearson correlation coefficient $(r=0.409)$ points to the existence of a significant positive and moderately strong association between Research and Development and SME growth and effectiveness. The researchers also established that successful Cameroonian SMEs often have some systems in place to monitor and control the quality of their products. Moreover, they periodically do formal or informal research exercises to keep abreast of their customer wants, needs and preferences. Research and development therefore remains an important ingredient for SME growth and effectiveness. Research and development is however not a cut-rate exercise. The Cameroonian government is advised to increase its research and development fund allocation to assisting SMEs with their research initiatives.

The purpose for any economic activity is to meet the needs of the market. SMEs that possess strong marketing skills were found to register more guaranteed growth and effectiveness over time than those who lack this important expertise. As shown in Table 3, SME growth and effectiveness is moderately and positively associated with good marketing management. This is demonstrated by a Pearson correlation coefficient, $r=$ 0.336. It is necessary therefore for owners and managers of SMEs to acquire the necessary skills that enable them to effectively utilize the essential marketing tools that help their SMEs meet its marketing objectives in any given target market. Such proper comprehension and acknowledgement of the importance of aspects as product, price, place, promotion, process, people, physical environment, and performance is necessary for any leader of a successful SME. 
Table 3 shows that variables such as the relevance of an entrepreneur's academic qualification, their budgeting ability, the SME's ability to provide product education to clients, availability of a business bank account, propensity of the business to own land, degree of competition, prevalence of crime and theft, all unveiled weak linear correlation with SME growth and effectiveness. While the majority of these variables have positive correlations, only the prevalence of crime and theft exhibited negative associations with the dependent variable.

The study also found that the entrepreneur's ability to budget increases the SMEs growth prospects. This is shown in Table 3 where the Pearson correlation coefficient 0.223 points to a positive relationship between the explanatory variable for budgeting capability and the SME growth and effectiveness. Budgeting helps the SMEs possess full control over their finances through the systematic planning of incomes, savings and expenditures. Once the SME maintains full control over their finances through proper budgeting, it becomes easier for them to set and track the direction towards which their business is going.

It is usually difficult to sell a product that is unknown to the market and it is the role of the SMEs staff to educate their clientele on their product offerings. This study discovered that SME's ability to provide product education to clients have a significant linear correlation with the SME growth and effectiveness. The Pearson's correlation coefficient $(r=0.206)$ suggests that SMEs who invested in educating customers about their products displayed better performance and also grew much bigger than those who did not teach clients.

While some SMEs do not utilize any banking services, this study established that it is beneficiary for Cameroonian entrepreneurs to have business bank accounts. Table 3 shows that there is a significant linear relationship between the independent variable for availability of a business bank account and the dependent variable. The Pearson's correlation coefficient $(r=0.230)$ indicate that SMEs who had a business bank account had better chances of growing over time and they were likely to operate more effectively than those without effective access to any banking services. It is therefore crucial for SMEs not to only own but also utilize their business bank accounts this can help the them keep their business finances safe. Separating personal finances from those of the business not only eases the allotting of funds for specific business related objectives but also simplifies processes like taxation. An SME that operates a business bank account has better access to mammoth bank loans that can be used for growth and expansion of the business venture.

The study found a weak but positive linear correlation between land ownership by SMEs and them being able to grow or at least operate effectively. What this implies is that, while it may be beneficiary for a business to own land in Cameroon, it is not a prerequisite for success since other SMEs still prospered while working on rented or leased properties. The Pearson's correlation coefficient $(r=0.235)$ suggests that most SMEs who operated on their own land in most cases had better chances to grow as compared to those who had less security of tenure. Having a fixed aboard is usually important for the business since customers can effortlessly identify your services with your permanent location. In other words clients can easily know where to find you in town and this is good for referrals.

Empirical evidence shown in Table 3 suggests that this competition is actually good for the growth and effectiveness of SMEs. The Pearson's correlation coefficient $(r=0.241)$ shows that the higher the competition in the market, the more SME operators are forced to think outside of the box and such innovativeness brings forth growth and innovation in the long run and thus effectiveness. It is however crucial that the government should ensure that the economy offers a fair and healthy competitive business environment that promotes not only the survival but the growth of SMEs. The study found out that some SMEs suffer fierce unfair competition particularly from the informal sector. Authorities should find ways of integrating the informal sector into the formal mainstream by for instance categorizing them as "big vs small" where big and influential informal traders may be registered and encouraged to adopt fair business practice.

According to this study's findings, the relevance of an entrepreneur's academic qualification as a variable portrays a significant and positive correlation with the dependent variable. This may mean that it is vital for an entrepreneur to acquire academic knowledge related to their enterprise's business. On the other hand, Table 3 displays a Pearson correlation coefficient of 0.203 that may be regarded as a weak linear correlation. This as a result may imply that though important, an entrepreneur's academic qualifications may in some instances differ from the scope of the SME business. In some cases, the study found some SMEs that still prospered not intrinsically because of their bosses' academic qualifications, but because of the management's special attributes such as their ability to learn fast, adaptability, enthusiasm, experience, amongst other factors.

While the occurrence of crime and theft displayed significant associations with the dependent variable, evidence in Table 3 shows that an increase in this variable will reduce the growth and effectiveness of SMEs in Cameroon. A number of SMEs who in the past were victims of crime and theft indicated that their progress was impinged greatly during their recovery phase from the subsequent losses of materials and property. Most of those who suffered this fate were SMEs in the primary and secondary sectors that sometimes lost their machinery, semi-finished or even some finished products while in storage. Though significant, the Pearson correlation coefficient $(r=-0.219)$ still points to a weak rapport between crime incidence and growth of SMEs 
Strengthening Enterprises Growth and Effectiveness in Developing Economies. A case of Very ..

thus showing that such unfortunate incidences are spasmodic. Nevertheless, law enforcement agents should continue working hard against perpetrators so as to encourage a more peaceful business environment where citizens and all economic players operate without fear of loss of property.

\section{Conclusion}

While the recipes for promoting SME growth and development may vary, this study accomplishes that the factors that are imperative for their success are generally of a socio-economic as well as a political nature. Concerned stakeholders in developing countries are urged to pay attention to upholding the entrepreneur's levels of education, boosting their experience, enhancing their financial management skills, and budgeting ability, curbing crime and corruption. Also crucial to the success of SMEs are skills like human resources management, administration, operations management, marketing management, research and development. Above and beyond these aspects, the government and all concerned parties are commended to make it their joint responsibility to create a favorable socio-economic and political environment that not only boosts enterprise confidence but also encourages more private investments into the economic system.

\section{Acknowledgements}

Our deepest gratitude goes to Yuzhen and the Bidja family, Glenda (nee Nyanhongo) Mandizvidza, Mukundinashe Thaniel Kudzai and Shylletti Nelia Tsivai for their support and inspiration.

\section{Journal Papers:}

\section{References}

[1]. Bouazza, A. B., Ardjouman, D., \& Abada, O. (2015). Establishing the Factors Affecting the Growth of Small and Medium-sized Enterprises in Algeria. American International Journal of Social Science, 4( 2), 101-115.

[2]. Chittithaworn, C., Islam, M. A., Keawchana, T., \& Yusuf, D. H. (2011). Factors Affecting Business Success of Small \& Medium Enterprises (SMEs) in Thailand. Asian Social Science, 7(5), 180-190.

[3]. Christina, B., Neelufer, A., \& Amri, S. A. (2014). Challenges and Barriers Encountered by the SMEs Owners in Muscat. International Journal of Small Business and Entrepreneurship Research, 2 (3), 1-13

[4]. Doh, S., \& Acs, Z. J. (2010). Innovation and social capital: a cross-country investigation. Industry \& Innovation, 17(3), 241 -262.

[5]. Doh, S., \& Kim, B. (2014). Government Support for SME innovations in the regional industries: The case of government financial support program in South Korea. Research Policy, 43, 1557-1569.

[6]. Haselip, J. A., Desgain, D., \& Mackenzie, G. A. (2014). Financing energy SMEs in Ghana and Senegal: Outcomes, barriers and prospects. Energy Policy, 65, 369-376.

[7]. Kamunge, M. S., Njeru, A., \& Tirimba, O. I. (2014). Factors Affecting the Perfomance of Small and Micro Entreprises in Limuru Town Market of Kiambu County, Kenya. International Journal of Scientific and Research Publications, 4(12), 1-20.

[8]. Mbugua, J. K., Mbugua, S. N., Wangoi, M., Ogada, J. O., \& Kariuki, J. N. (2013). Factors Affecting the Growth of Micro and Small Enterprises: A Case of Tailoring and Dressmaking Enterprises in Eldoret. International Journal of Business and Social Science, 4 (5), 285-293.

\section{Reports:}

[9]. Business in Cameroon. (2015). COP 21: Cameroon's commitments to preserve the planet. No. 33. Yaounde: Stratline Limited. Retrieved February 20, 2017, from http://www.businessincameroon.com/pdf/BC33.pdf

[10]. CFCE. (2015). Statistics of SME created from 2010-2014 through the CFCE. Yaounde, Cameroon: Centre for the Facilitation of Creation of Enterprises (CFCE)

[11]. GAN Integrity. (2015). Cameroon Corruption Report. Copenhagen, Denmark: GAN Intengrity Inc. Retrieved January 25, 2017, from http://www.business-anti-corruption.com/country-profiles/cameroon

Books:

[12]. DeVellis, R. F. (2012). Scale development: Theory and applications, $3^{\text {rd }}$ Edition. Los Angeles: Sage.

[13]. George, D., \& Mallery, P. (2003). SPSS for Windows step by step: A simple guide and reference 11.0 update (4th ed.). Boston: Allyn \& Bacon.

\section{Proceedings Papers:}

[14]. Afriland First Bank. (2015). Finance for All: Promoting Financial Inclusion in Central Africa, The Challenges of Financing SMEs. ECCAS Regional Conference, Brazzaville, Congo: IMF

[15]. Senderovitz, M. (2009). How are SMEs Defined in Current Research? Proceedings of AGSE. Adelaide, Australia: AGSE.

Websites:

[16]. Lund, A., \& Lund, M. (2013). Pearson Product-Moment Correlation. UK: Lund Research Ltd . Retrieved January 28, 2017, from https://statistics.laerd.com/statistical-guides/pearson-correlation-coefficient-statistical-guide.php 